\title{
Uptake of Liposomally Entrapped Fluorescent Antisense Oligonucleotides in NG108-15 Cells: Conventional versus pH-Sensitive
}

\author{
Natasa Skalko-Basnet, Michihisa Tohda, and Hiroshi Watanabe* \\ Department of Pharmacology, Institute of Natural Medicine, Toyama Medical and Pharmaceutical University; 2630 \\ Sugitani, Toyama 930-0194, Japan. Received August 2, 2002; accepted September 13, 2002
}

\begin{abstract}
Antisense oligodeoxynucleotides (asODN) are novel therapeutic agents designed to alter RNA metabolism, ultimately resulting in decreased production of disease-associated gene products. To investigate internalisation of liposomally delivered asODN in NG108-15 cells, a hybrid cell line of mouse neuroblastoma and rat glioma, and assure that uptake of marker corresponds to that of antisense, we compared the cellular uptake of fluorescently labelled marker (fluorescein isothiocyanate (FITC)-dextran) and antisense oligonucleotide (FITC-asODN), entrapped either in conventional soy phosphatidylcholine (SPC) liposomes or pH-sensitive liposomes (composed of dioleoylphosphatidylethanolamine and cholesteryl hemisuccinate in a molar ratio of $3: 2$ ). Both SPC and pHsensitive liposomes were prepared by a modified freeze-thawing method. Entrapment efficiencies (about $20 \%$ of the original material) did not depend on the liposome compositions and fluorescent material used. Fluorescence activated cell sorting (FACS) analysis was used to quantify the association of fluorescent material with the NG108-15 cells, whereas confocal microscopy gave insight on the location of cell associated-fluorescence. Conventional liposomes failed to deliver fluorescent material into the cells, but in contrast, $\mathrm{pH}$-sensitive liposomes significantly improved the uptake of both FITC-dextran and FITC-asODN, with the uptake of liposomal FITC-dextran being greater than the uptake of liposomal FITC-asODN. These results suggest that pH-sensitive liposomes can be applied as a carrier system in the delivery of genetic material into the cells.
\end{abstract}

Key words pH-sensitive liposome; conventional liposome; antisense oligonucleotide; NG108-15 cell; fluorescence activated cell sorting; confocal microscopy

Antisense oligodeoxynucleotides (asODN) are becoming a focus of attention as a tool for down-regulation of gene expressions that contribute to disease states. Intense efforts to develop and exploit antisense technology over the past decade have resulted in the first drug based on antisense technology approved by FDA (Vitravene). However, in spite of some successful antisense applications, there are still many unanswered questions regarding antisense concept and the therapeutic potential of the technology. ${ }^{1)}$ The progress in the field is limited by two main obstacles, one being the finding of an accessible sequence for an asODN to hybridise with its mRNA target, and the second the ability to deliver asODN into cells and have them reach their target. ${ }^{2)}$ The mechanism by which oligonucleotides enter the cells remains controversial. Different cells widely differ in the uptake mechanism, therefore, it is of great importance to observe cell-staining patterns obtained after transfection with fluorescently conjugated oligonucleotides. ${ }^{3)}$ As more antisense therapeutics are proven to be effective and become commercially successful, more attention must be paid to delivery systems for antisense. By applying the delivery system, it is possible to increase the cellular uptake of antisense and reduce the dose needed for the antisense effect. Liposomes are a protective biocompatible and biodegradable delivery system that can enhance cellular uptake. Although conventional liposomes are able to protect active molecules from degradation and improve their cellular accumulation, once in cells, the entrapped material is mostly degraded within the lysosomes. In that case, the liposome content will be released in a subcellular environment where it can be degraded and the delivery will occur in the lysosomes, preventing oligonucleotides to reach the mRNA target. ${ }^{4)}$ Interaction with lysosomes results in the degradation of all types of ODNs. Circumventing the endosomal/lysosomal pathway has been suggested to be one mechanism by which to enhance the delivery and efficacy of antisense compounds. $\mathrm{pH}-\mathrm{Sensitive} \mathrm{liposomes} \mathrm{were}$ designed to circumvent delivery of liposome content to the lysososmes of cells following the internalisation of the vesicle via the endocytic pathway. $\mathrm{pH}-$ Sensitive liposomes destabilise membranes or become fusogenic when they are exposed to an acidic environment. They might transfer its contents into the cytoplasm before the liposome can be conveyed to the lysosomes. ${ }^{5)}$ In the majority of $\mathrm{pH}$-sensitive compositions, a lipid containing a $\mathrm{pH}$ titratable group is mixed with phosphatidylethanolamine containing unsaturated acyl chains. ${ }^{6}$ Among them, dioleoylphosphatidylethanolamine/ cholesteryl hemisuccinate (DOPE/CHEMS) composition seems to be the most efficient liposomal composition. ${ }^{7)}$

An increase in the cellular uptake of $\mathrm{pH}$-sensitive liposomally delivered material have been proved in the various cells in in vitro conditions. ${ }^{8-12)}$ Due to the high cost of asODN, in many experiments, fluorescently labelled marker of similar molecular weight was/is used to mimic the antisense molecule. In order to determine how comparable are the actual uptake of marker and the uptake of antisense molecule, we investigated the association of conventional and $\mathrm{pH}-$ sensitive liposomes carrying either fluorescent marker or fluorescent asODN with the NG108-15 cells. Cell-associated fluorescence was determined by fluorescence activated cells sorting (FACS analysis) and confocal microscopy.

\section{MATERIALS AND METHODS}

Materials Cholesteryl hemisuccinate (CHEMS), dioleoylphosphatidylethanolamine (DOPE), fluorescein isothiocyanate (FITC)-dextran (Mw 4400), sodium cholate and SPC (soy phosphatidylcholine) were purchased from Sigma (St Louis, MO, U.S.A.). Oligonucleotide with a sequence $5^{\prime}$ - 
ATT GAA ACA AGC GTC CAC CAT CGG AG-FITC (Mw 7973.3 containing $33.3 \%$ adenine, $25.9 \%$ cytosine, $22.2 \%$ guanine, $14.8 \%$ thymine and $3.7 \%$ FITC) was the product of ESPEC (Tskuba, Japan). The NG108-15 cells were the generous gift from Professor Higashida, Dept. of Biophysical Genetics, Molecular Medicine and Bioinformatics, Kanazawa University Graduate School of Medicine, Japan. HEPES [( $N$-(2-hydroxyethyl)piperazine- $N{ }^{\prime}$-(2-ethanesulfonic acid)] was from Dojin (Kumamoto, Japan). All other chemicals were of analytical grade.

Liposome Preparation Liposomes were prepared by a modified freeze-thawing method. ${ }^{12}$ In brief, lipid films ( $50 \mathrm{~mm}$ total lipid) were prepared by evaporation of either the solutions of DOPE (in chloroform) and CHEMS (in tetrahydrofuran/methanol $2: 1 \mathrm{v} / \mathrm{v}$ ) in the molar ratio of $3: 2$ in the case of $\mathrm{pH}$-sensitive liposomes, or SPC (in chloroform) for SPC liposomes. To the dry film (after vacuum desiccation) $0.5 \mathrm{ml}$ HEPES (10 mm with $150 \mathrm{~mm} \mathrm{NaCl}$; $\mathrm{pH} 7.4)$ containing either FITC-dextran $(5 \mathrm{~mm})$ or FITC-asODN $(100 \mu \mathrm{M})$ was added, the suspension vigorously shaken, probe sonicated $8 \times 30 \mathrm{~s}$ (with resting periods of $30 \mathrm{~s}$ ) and left at room temperature for $30 \mathrm{~min}$. The suspension was rapidly frozen in liquid nitrogen, than left at room temperature to thaw completely, for total of three times. For adsorption experiments, control (empty) liposomes (50 mM total lipid) were prepared under the same conditions and incubated overnight (at $4{ }^{\circ} \mathrm{C}$ ) with either free FITC-dextran or FITC-asODN (in the same concentrations as used in entrapment experiments). Gel chromatography on a Sepharose CL-4B (Amersham Pharmacia, Buckinghamshire, U.K.) gel column was applied to separate nonliposomal material from liposomally-associated material. The entrapment efficiencies were determined fluorimetrically. The mean diameter of all preparations was between 250 $300 \mathrm{~nm}$ as measured by Olympus Provis AX 80 microscope (Japan) connected with an image analyser system (C Imaging Systems, Neuroscience Inc, Osaka, Japan).

Cellular Experiments The NG108-15 cell line was cultured in Dulbecco's modified Eagle's medium (DMEM) containing HAT $(100 \mu \mathrm{M}$ hypoxantine, $1 \mu \mathrm{M}$ aminopterin and $16 \mu \mathrm{m}$ thymidine) supplemented with $5 \%$ fetal calf serum (FCS). The cells $\left(5 \times 10^{4}\right.$ cells per well) were seeded into $24-$ well Falcon culture plates (Becton Dickinson, Franklin Lakes, NJ, U.S.A.) prior to experiments and maintained at $37^{\circ} \mathrm{C}$ in a humidified atmosphere $\left(10 \% \mathrm{CO}_{2}\right)$. In a typical experiment, the medium was removed from the well containing cells and cells incubated with either liposomes (SPC or $\mathrm{pH}$-sensitive) containing FITC-dextran or FITC-asODN or free FITC-material (all preparations in DMEM containing $1 \% \mathrm{FCS} ; 0.5 \mathrm{ml}$ per well) for certain time intervals. After removing the media and liposomes, the cells were washed with PBS and centrifuged $(5 \mathrm{~min}$ at $1200 \times \boldsymbol{g})$. The pellets were resuspended in PBS $(0.5 \mathrm{ml})$, stored on ice and used for FACS analysis (within $30 \mathrm{~min}$ ). As a control, the cells were incubated with HEPES buffer and subjected to the same treatment as the cells incubated with either free FITC-material or liposomal preparations.

FACS Analysis FACS analysis was performed on a FACS sorter (Beckton-Dickinson, Heidelberg, Germany) and the percentage of cell-associated fluorescence assessed. 10000 individual cells were detected by stray light and the percentage of cells which were additionally fluorescent was calculated by CellQuest software (Beckton-Dickinson, Heidelberg, Germany).

Confocal Microscopy Liposomes (both SPC and $\mathrm{pH}-$ sensitive) containing FITC-dextran or FITC-asODN, as well as FITC-dextran or FITC-asODN in a free form were incubated for certain time intervals at $37^{\circ} \mathrm{C}$ (as described earlier) with NG108-15 cells seeded on Falcon (culture slides (Beckton Dickinson, U.S.A.)). The cells were washed with PBS and fixed with formaldehyde (10\%) for $25 \mathrm{~min}$ at room temperature. The slides were embedded in an anti-fading medium (VectraShield ${ }^{\mathbb{B}}$, Vector Laboratories, U.S.A.) and fixed on cover slips. Samples were examined by an Olympus LSM-GB 200-ITM-2 laser scanning confocal microscope (Tokyo, Japan).

\section{RESULTS}

Entrapment of Fluorescent Material in Liposomes In order to serve as a delivery system and increase the cellular uptake of any molecules, liposomes are expected to entrap sufficient amount of the material destined to be entrapped. The liposome preparation method applied in our experiments enabled the entrapment of up to $20 \%$ of the material taken into the preparation (Table 1). The average size of liposomes was $250-300 \mathrm{~nm}$, regardless of the lipid composition and entrapped material, and was dependent on the conditions used in preparing the liposomes (data not included). No differences between entrapment efficiencies between liposomes made of SPC and those made of $\mathrm{pH}$-sensitive lipids were observed. The actual entrapment was not dependent on the type of fluorescent material used in experiments (Table 1). To ensure that liposomally-associated material was entrapped and not adsorbed onto liposomal surface, we performed control experiments in which empty liposomes were incubated with free FITC-dextran or FITC-asODN, separated by gel chromatography and liposome-associated fluorescence determined fluorimetrically. The adsorption was below $1 \%$ of the fluorescent material taken into incubation. In that way, we ensured that liposomally-associated material was indeed entrapped within liposomes.

Liposomes-Cells Association Conventional SPC liposomes failed to increase the cellular uptake of FITC-labelled material (Fig. 1). Even for very high lipid concentrations (over $1 \mu \mathrm{mol}$ per $5 \times 10^{4}$ cells) the uptake of entrapped material was bellow $20 \%$. On the contrary, $\mathrm{pH}$-sensitive liposomes significantly improved (Student-Newman-Keuls test after two way analysis of variance (ANOVA)) the cellular association

Table 1. Entrapment of Fluorescently Labelled Material in Liposomes

\begin{tabular}{llcc}
\hline \hline $\begin{array}{c}\text { Lipid } \\
\text { composition }\end{array}$ & $\begin{array}{c}\text { Fluorescent } \\
\text { material }\end{array}$ & $\begin{array}{c}\text { Entrapment } \\
\text { efficiency (\%) }\end{array}$ & $\begin{array}{c}\text { Control } \\
\text { (adsorption; \%) }\end{array}$ \\
\hline SPC & FITC-dextran & $17.9 \pm 2.4$ & $0.3 \pm 0.2$ \\
& FITC-asODN & $19.5 \pm 1.2$ & $0.9 \pm 0.1$ \\
$\mathrm{pH}$-sensitive & FITC-dextran & $20.1 \pm 1.8$ & $0.5 \pm 0.2$ \\
& FITC-asODN & $19.9 \pm 0.8$ & $0.9 \pm 0.3$ \\
\hline
\end{tabular}

Liposomes (average mean diameter between $250-300 \mathrm{~nm}$ ) entrapping either FITCdextran or FITC-asODN were prepared by a modified freeze-thawing method (for details see Materials and Methods). For the control experiments, empty liposomes were incubated with FITC-dextran or FITC-asODN (in amount as used for the entrapment). Unentrapped or nonadsorbed material was separated from liposomes by gel chromatography. Entrapment and adsorption percentages were measured fluorimetrically. The values denote the mean of three preparations \pm S.D. 


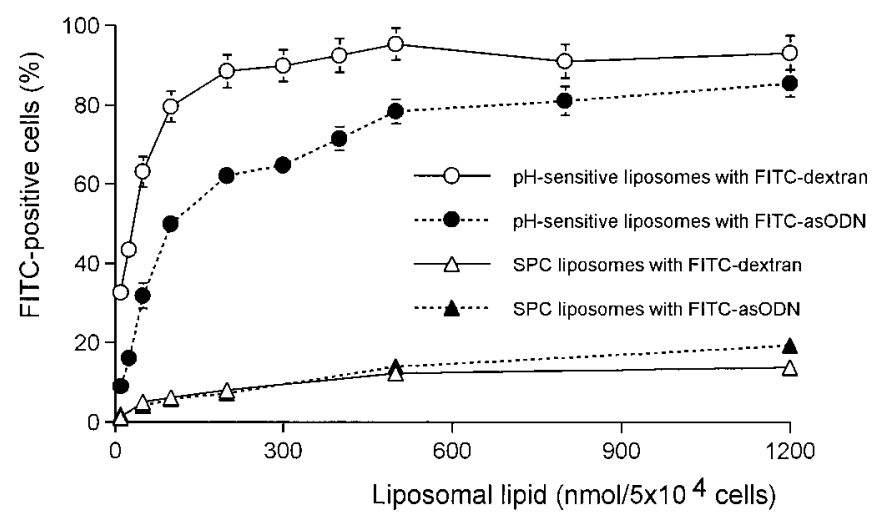

Fig. 1. Cellular Uptake of Liposomally Delivered Fluorescent Material by NG108-15 Cells

SPC and $\mathrm{pH}$-sensitive liposomes were prepared as described in the Methods. Liposomes containing either FITC-dextran or FITC-asODN were incubated with the NG108-15 cells for $2 \mathrm{~h}$ at $37^{\circ} \mathrm{C}$. After washing, the cells were subjected to FACSanalysis. The values denote the mean of three separate experiments \pm S.D.

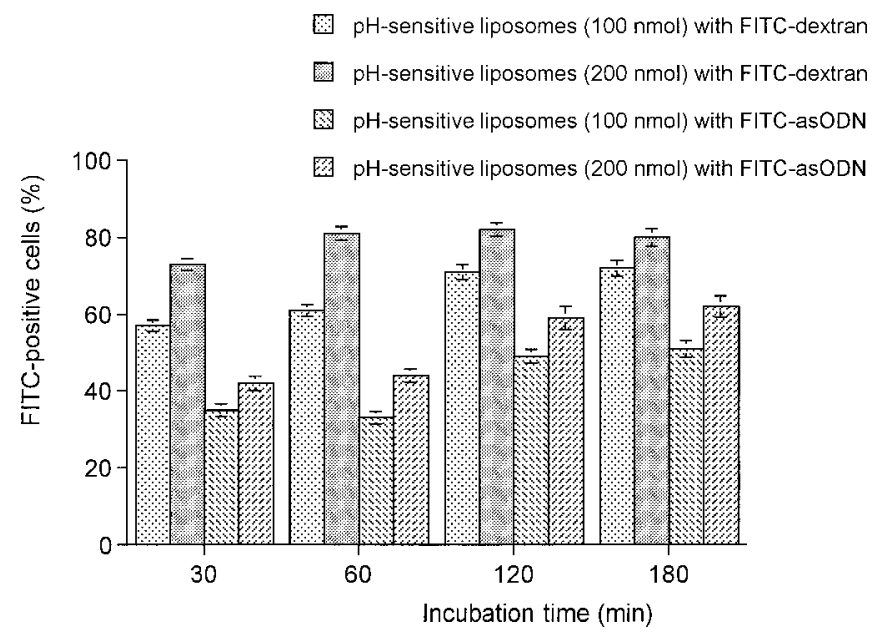

Fig. 2. The Effect of Incubation Time for pH-Sensitive Liposomes Entrapping Either FITC-Dextran or FITC-asODN (100 and 200 nmol Liposomal Lipid per $5 \times 10^{4}$ Cells) Incubated with the NG108-15 Cells for Certain Time Intervals (for Details see Materials and Methods) and Subjected to FACS Analysis

The values denote the mean of three separate experiments \pm S.D.

of entrapped material. For FITC-dextran entrapped in pHsensitive liposomes, the uptake was concentration-dependent reaching a saturation plateau at $300 \mathrm{nmol}$ of liposomal lipid per $5 \times 10^{4}$ cells $(89 \%$ of all treated cells were FITC-positive). Although the uptake of FITC-asODN delivered by $\mathrm{pH}-$ sensitive liposomes was also concentration-dependent, the saturation plateau was reached at higher lipid concentration $(500 \mathrm{nmol})$ and the total uptake of liposomally entrapped fluorescent antisense was lower (Fig. 1). The cell viability seemed to be not changed even at $500 \mathrm{nmol}$. When this NG108-15 cells died, the cells detached from dishes. The amount of floated and attached cells seemed to be not changed, although cell shape changed to be globular at high concentration. At the same time, free FITC-dextran (control) was taken up by only $2.4 \%$ of the cells, whereas free FITC-asODN was taken up by $23 \%$ of the cells (the amount of free FITC-dextran or FITC-asODN incubated with the cells corresponded to that entrapped in $300 \mathrm{nmol}$ liposomal lipid; data not shown). Considering that the uptake of free
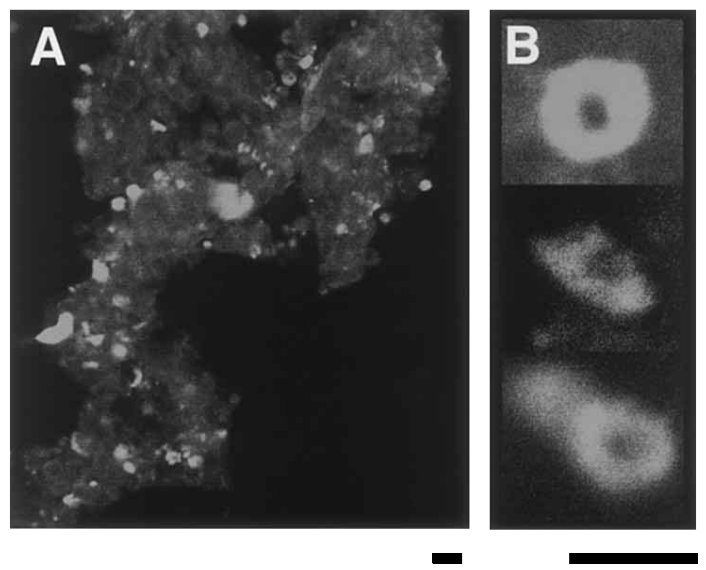

Fig. 3. Representative Confocal Micrographs of the NG108-15 Cells after $2 \mathrm{~h}$ Incubation with $\mathrm{pH}$-Sensitive Liposomes Entrapping FITC-asODN (A) and of the Individual Cells (B)

For the details see the Methods. The bar represents $20 \mu \mathrm{m}$.

FITC-asODN (nonliposomal) was more than 20\%, SPC liposomes clearly failed in improving the delivery of the entrapped antisense into NG108-15 cells. Therefore, time-dependency of cell-association experiments were performed with $\mathrm{pH}$-sensitive liposomes. The effect of incubation time on the cells-liposomes interaction is presented in Fig. 2. The cells incubated with liposomally entrapped FITC-material (two lipid concentrations, 100 and $200 \mathrm{nmol}$ lipid $/ 5 \times 10^{4}$ cells, respectively) for certain time intervals $(30 \mathrm{~min}, 1 \mathrm{~h}, 2 \mathrm{~h}$, $3 \mathrm{~h}$ ) showed that the maximum association between fluorescent material and the cells was achieved already after $2 \mathrm{~h}$ incubation both in the case of liposomal marker and liposomal antisense, as well as for both lipid concentrations. Concentration-dependency of liposomes-cells association was confirmed. No further improvement in uptake was detected in cells incubated with liposomes for $3 \mathrm{~h}$, therefore, in confocal experiments, cell-associated fluorescence was examined after $2 \mathrm{~h}$ incubation.

Confocal Microscopy Figure 3 represents the typical confocal images of the NG108-15 cells after $2 \mathrm{~h}$ incubation with $\mathrm{pH}$-sensitive liposomes ( $200 \mathrm{nmol}$ lipid per $5 \times 10^{4}$ cells) carrying FITC-asODN (Fig. 3A). The cell-associated fluorescence appeared to be evenly spread through the cells, indicating that liposomes delivered the entrapped material into the cytoplasm (Fig. 3B). The cytoplasmic location of cell-associated fluorescence suggests that lysosomal degradation of fluorescent material was avoided.

\section{DISCUSSION}

The use of antisense oligonucleotides represents a novel technology that shall be applied in gene therapy. ${ }^{3)}$ However, in spite of the growing number of research laboratories involved in the field, some of the problems such as distribution, cellular uptake and cost remain to be solved. ${ }^{13)}$ Antisense molecules bind specifically to a target mRNA via WatsonCrick base pair interaction and block the translation of corresponding protein. For this to occur, antisense must penetrate the cell membrane and achieve the appropriate concentration in the correct intracellular compartment (cytoplasm or nucleus). ${ }^{14)}$ The cellular uptake of $\mathrm{pH}$-sensitive liposomal 
marker was higher than the uptake of liposomal antisense (Fig. 1). In both cases, the saturation of the uptake was observed, but for liposomal antisense saturation occurred at higher lipid concentration than for the marker. Important difference in uptake level is the fact that, in contrast to free marker which was not taken up by the cells, free antisense oligonucleotides were taken up by the cells. As previously shown, the uptake of free antisense was concentration- and time-dependent. ${ }^{12)}$ Internalisation experiments of either free fluorescently labelled or liposomally entrapped material are best performed by FACS analysis, a method that has an advantage that dead cells are eliminated from the final analysis which is important because non viable cells internalise significantly more asODN than viable ones. FACS analysis can precisely and reproducibly determine the amount of cell-associated fluorescence, however, for the actual localisation of the fluorescent molecules in the cells, another additional techniques such as confocal microscopy are necessary. The localisation of cell associated fluorescence was in cytoplasm (Fig. 3B) and no differences in the cellular localisation between liposomally delivered FITC-asODN and FITC-dextran were detected (images not shown). pH-Sensitive liposomes appeared to successfully deliver entrapped material into cells cytoplasm. It is reasonable to expect that the amount of antisense oligonucleotides taken up by the cells, and its cytoplasmic location, would enable antisense to perform their specific action in the cells. In spite of many similarities in the uptake of liposomal marker and antisense, one should be aware that the uptake cannot be always directly compared, especially since free antisense molecules will be taken up by the cells as well (at least by neuroblastoma cells). However, it is expected that oligonucleotides unassisted by a carrier can produce antisense activity only occasionally and in an uncontrollable fashion. They must remain intact to be effective and by the use of a suitable carrier system dose needed for their action can be reduced. Prior to transfection experiments, it is of a great importance to clarify and establish the parameters important for the uptake process. A prerequisite for the transfection to occur is delivery of genetic material into and through cells. As in many experiments fluorescently labelled marker of similar molecular weight is/was used to mimic/replace the actual antisense oligonucleotide, it is important to assure that the uptake pattern of liposomally entrapped marker corresponds to that of liposomally entrapped antisense. Therefore, we performed in vitro studies and directly compared cell-associated fluorescence of liposomally delivered marker with that of liposomally delivered antisense. Control (adsorption) experiments, in which empty liposomes were incubated with free antisense oligonucleotides, were performed to assure and confirm that no interactions between the negative charge of the oligonucleotides and the positive charge of the DOPE amino group occurred, and that oligonucleotides were indeed entrapped within liposomes (Table 1). Our findings are in an agreement with the work of DeOliviera et al. ${ }^{15}$ Antisense oligonucleotides are internalised and distributed into cells in a time-, temperature-, concentration-, sequences- and cell line dependent manner. The cellular uptake mechanism of antisense oligonucleotides, although still controversial, is proposed to be receptor-mediated. ${ }^{16)}$ In an elegant set of experiments, Beltinger et al. ${ }^{17)}$ showed that endocytosis, mediated by a re- ceptor-like mechanism, was predominant cellular uptake mechanism at asODN concentrations below $1 \mu \mathrm{M}$, whereas fluid-phase endocytosis prevailed at higher concentrations.

We are aware of the fact that the generalisation of in vitro experiments is virtually impossible, as it is well established that the cell type has a dramatic effect on total uptake, kinetic of the uptake and pattern of subcellular distribution of antisense molecule. ${ }^{1,18)}$ Therefore, our findings can only be applied to neuroblastoma cells. One further step forward in site-specific delivery would be targeting of carrier entrapping antisense molecules, as recently proven by Pagnan et al. ${ }^{19}$ By applying targeted liposomes, the concentration needed for the antisense action was reduced by several folds. Although the liposome composition applied in their experiments was different than our $\mathrm{pH}$-sensitive composition, coupling of the ligands to the surface of $\mathrm{pH}$-sensitive liposomes has already been established. ${ }^{8)}$ Targeted $\mathrm{pH}$-sensitive liposomes could further improve cellular uptake of antisense oligonucleotides.

In conclusion, $\mathrm{pH}$-sensitive liposomes significantly improved the cellular uptake of entrapped material and have been proven to be suitable carrier system in delivery to neuroblastoma cells. When applying the fluorescently labelled marker as a replacement for antisense, one should bear in mind that the cellular uptake of liposomal marker and liposomal antisense cannot be always comparative, especially when free antisense oligonucleotides penetrate the cells as well. The specific action of antisense molecules (both free and liposomally entrapped) in the cells remains to be investigated, both in in vitro and in vivo conditions.

Acknowledgements This work was in part supported by a Grant-in-Aid for Scientific Research (B) to Dr. M. Tohda (\#124770194) and a Grant-in-Aid for Learning Research Utilizing Potential Science \& Technology to Dr. H. Watanabe form the Ministry of Education, Culture, Sports, Science and Technology, Japan.

\section{REFERENCES}

1) Crooke S. T., Methods Enzymol., 313, 3-45 (2000).

2) Gewirtz A. M., Stein C. A., Glazer P. M., Proc. Natl. Acad. Sci. U.S.A., 93, 3161-3163 (1996).

3) Myers K. J., Dean N. M., Trends Pharmacol. Sci., 21, 19-23 (2000).

4) Fattal E., Delattre J., Dubernet C., Couvreur P., STP Pharm. Sci., 9, 383-390 (1999).

5) Couvreur P., Fattal E., Malvy C., Dubernet C., J. Liposome Res., 7, 118 (1997).

6) Chu C.-J., Szoka F. C., J. Liposome Res., 4, 361-395 (1994).

7) Chu C.-J., Dijkstra J., Lai M-Z., Hong K., Szoka F. C., Pharmacol. Res., 7, 824-834 (1990).

8) Skalko N., Peschka R., Altenschmidt U., Lung A., Schubert R., FEBS Lett., 434, 351-356 (1998).

9) Tachibana R., Harashima H., Shono M., Azumano M., Niwa M., Futaki S., Kiwada H., Biochem. Biophys. Res. Commun., 251, 538-544 (1998).

10) Peschka-Suss R., Skalko-Basnet N., J. Liposome Res., 10, 43-59 (2000).

11) Lubrich B., van Calker D., Peschka-Suss R., Eur. J. Biochem., 267, $2432-2438$ (2000).

12) Skalko-Basnet N., Tohda M., Watanabe H., Neuroreport, 11, 31173121 (2000).

13) Miller K. J., Das S. K., Pharm. Sci. Technol. Today, 1, 377-386 (1998).

14) Benimetskaya L., Tonkinson J., Stein C. A., Methods Enzymol., 313, $287-297$ (2000). 
15) DeOliveira M. C., Fattal E., Ropert C., Malvy C., Couvreur P., J. Control. Release, 48, 179-184 (1997).

16) Crooke R. M., Graham M. J., Cooke M. E., Crooke S. T., J. Pharmacol. Exp. Ther, 275, 462- 473 (1995).

17) Beltinger C., Saragovi H. U., Smith R. M., LeSauter L., Shah N., DeDionisio L., Christensen L., Raible A., Jarett L., Gewirtz A. M., J. Clin. Invest., 95, 1814-1823 (1995).
18) Lai J., Crook T. J., Payne A., Lynch R. M., Porreca F., J. Pharmacol. Exp. Ther., 281, 589-596 (1997).

19) Pagnan G., Stuart D. D., Pastorino F., Raffaghello L., Montaldo P. G., Allen T. M., Calabretta B., Ponzoni M., J. Natl. Cancer Inst., 92, 253-261 (2000). 